\title{
Therapeutic Potential of Adenosine Transport Modulators for Cardiovascular Protection
}

\section{Shyam Sundar Kolathuru and Pollen K Yeung*}

College of Pharmacy and Department of Medicine, Dalhousie University, Canada

\section{Introduction}

Cardiovascular disease including stroke is the leading cause of death and disability worldwide [1] and an enormous economic burden to our societies [2]. Based on the latest statistics released for heart and stroke disease, an estimated 83,600,000 adults in the United State (US) (>30\%) have one or more types of cardiovascular disease (CVD) of whom more than $90 \%$ have hypertension, $18 \%$ have coronary heart disease (CHD), close to $10 \%$ have myocardial infarction (MI) and $8 \%$ have stroke. The total direct and indirect cost in the US alone for treatment of cardiovascular diseases (hospitalization, drugs, home healthcare, etc.) and loss of productivity and morbidity is estimated at close to $\$ 315$ billion US per year [3]. Thus prevention by improving diagnosis and drug treatment strategies could provide a huge saving for the health care cost worldwide.

The importance of adenosine in regulating many biological functions has long been recognized, especially for their effects on the cardiovascular system $[4,5]$. It is known that adenosine metabolism is a key factor in regulation of coronary blood flow [6], inhibiting platelet aggregation [7,8] protection of myocardium [9], neuromodulation [10], attenuating tissue necrosis [11], ischemic preconditioning [12], immunomodulation [13], energy metabolism [14], and pain mediation [15] which maintain the homeostasis of the cardiovascular system. Adenosine modulates these actions by interacting with adenosine receptors (AR), which are distributed ubiquitously throughout the body [16]. It is believed that metabolic condition of the myocardium may be assessed quantitatively by the level of adenosine production which maintains a healthy balance between energy supply and demand in the cardiovascular system [17].

Under pathologic and stressful conditions such as those associated with ischemia, hypoxia, trauma, seizure, inflammation and pain, there is an increased demand for energy triggering an increased catabolism of ATP to AMP and subsequently to adenosine, resulting in elevated levels of adenosine in both extracellular and intracellular space which protects against tissue damage [18]. There have been many studies both in animal models and humans to explore the cardiovascular protective effects of adenosine in the past few decades. These studies have laid the ground work and set the pace for future research on adenosine, adenosine agonists and adenosine re-uptake inhibitors as cardiovascular protective agents.

\section{Adenosine production and metabolism under normal physiological and hypoxic conditions in the cardiovascular system}

Under normal physiological conditions the main source of adenosine is from catabolism of ATP to ADP and then to AMP, which is further catabolized by ecto and endo 5 'nucleotidase to produce adenosine [19]. Another source of adenosine is from hydrolysis of S-adenosylhomocystein which is derived from the transmethylation pathway utilizing S-adenosylmethionine [20]. Intracellular adenosine can undergo rephosphorylation to form AMP and other adenine nucleotides by adenosine kinase [21] or metabolized to inosine by adenosine deaminase respectively [22], which maintain low concentrations of adenosine under basal or normal physiologic condition [19]. However, during ischemia/hypoxia or in extremely heavy workloads, there is an increased demand of energy which triggers a breakdown of ATP and release of adenosine locally and into systemic circulation [23]. The adenosine released is taken up rapidly by endothelial cells and red blood cells via nucleoside transporters and subsequently metabolized to ATP [24] to maintain the low basal level of adenosine.

\section{Importance of ATP synthase and adenylate kinase in cardioprotection}

ATP synthase is a mitochondrial enzyme involved in oxidative phosphorylation during ATP synthesis [25]. It is a key enzyme directly involved in cellular energy metabolism and the widely conceived theory of ischemia preconditioning and a target for cardioprotection [26,27]. Pharmacologic agents which activate ATP synthase or maintain its integrity in adversary conditions such as demonstrated for the tetracyclines [28] may help to preserve intracellular ATP in ischemia. On the other hand, adenylate kinases are abundant phosphotransferases catalyzing the inter-conversion of adenine nucleotides and thus regulate the adenine nucleotide ratios in different intracellular compartments. The enzymes are present ubiquitously in various tissues in the body including the RBC [29], and involved in regulating energy metabolism and energy signaling in the cardiovascular system as well as in the body $[30,31]$. Thus both enzymes are critically important working together to maintain an optimum balance of energy supply and demand for cardiovascular homeostasis.

\section{Adenosine transport modulators for cardioprotection}

It is clear that adenosine plays a pivotal role in cardioprotection not only by its receptor mediated actions, but also as a source of supply for intracellular AMP [9] which is a substrate as well as an important signaling mechanism for ATP synthesis [26]. It has been reported that inhibiting nucleoside transporter by R75231 improves the functional recovery from ischemia/reperfusion injury in an isolated rabbit heart model [32]. Similarly, dipyridamole and other nucleoside transporter inhibitors (NTI) such as dilazep have been shown to be protective in various animal models of myocardial ischemia, which may be mediated via adenosine $A(1)$ receptor [33]. It was suggested that the order of

*Corresponding author: Pollen K Yeung, Professor of Pharmacy and Medicine Dalhousie University, Halifax, NS, Canada,Tel: 902-4943845; Fax: 902-4941396; E-mail: Pollen.Yeung@Dal.Ca

Received May 13, 2015; Accepted May 14, 2015; Published May 20, 2015

Citation: Kolathuru SS, Yeung PK (2015) Therapeutic Potential of Adenosine Transport Modulators for Cardiovascular Protection. Cardiol Pharmacol 4: e127. doi:10.4172/2329-6607.1000e127

Copyright: ( 2015 Yeung PK et al. This is an open-access article distributed under the terms of the Creative Commons Attribution License, which permits unrestricted use, distribution, and reproduction in any medium, provided the original author and source are credited. 
Citation: Kolathuru SS, Yeung PK (2015) Therapeutic Potential of Adenosine Transport Modulators for Cardiovascular Protection. Cardiol Pharmacol 4: e127. doi:10.4172/2329-6607.1000e127

Page 2 of 3

potency inhibiting adenosine uptake by erythrocytes is draflazine $\geq$ dilazep $>$ R $75231>$ NBMPR $>$ mioflazine $=$ dipyridamole $>$ lidoflazine [34]. Using mammalian cardiac myocytes, it was shown that adenosine attenuated the stimulatory effects of isoproterenol via blockade of calcium influx [35], which may explain at least partly the cardiovascular protective effect of calcium channel blockers [36], although these agents may also have a direct effect modulating the transport and metabolism of adenosine [37-39].

\section{Future Direction}

The role of adenosine in cardiovascular protection in ischemia and reperfusion injury is well established. Pharmacologic agents such as the NTI have been used successfully in animal models and patients to increase extracellular adenosine concentrations and enhance the protective effect of endogenous adenosine. While adenosine kinase which metabolizes adenosine to adenosine monophosphate (AMP) has been advocated as a target for drug development for cardiovascular and metabolic diseases [21], the adenosine transporters, ATP synthase as well as adenylate kinase may also be exploited as drug targets to develop selective agents for cardiovascular prevention and protection. A better understanding and refinement of adenosine transporters and these energy metabolism enzymes can improve the safety and efficacy of the NTI as a therapeutic strategy for management of ischemic heart disease and related cardiovascular conditions. While most of the earlier studies have focused on the effect of NTI on extracellular adenosine or adenosine metabolism in cardiac myocytes, little is known of the effect of these agents on ATP/adenosine metabolism in erythrocytes (RBC) in vivo under normal or in ischemia conditions. It is probable that the increase in plasma concentrations of adenosine during ischemia could be attributed at least in part to metabolic breakdown of ATP in the RBC [40]. Blockade of the reuptake of adenosine by the NTI could moderate ATP metabolism and the nucleotide pool in the RBC which may be an important factor affecting the safety and efficacy of these therapeutic agents. The relationship between the nucleotide concentrations in the $\mathrm{RBC}$ and those of the endothelium and myocardium, and the potential of ATP metabolism in the RBC as a surrogate biomarker for energy balance in the body warrants further studies. Understanding these concepts and the inter-play of ATP metabolism in these different tissues would enable us to explore the future potential of NTI and other modulators of the nucleoside transporters for management of myocardial ischemia.

\section{References}

1. Roger Veronique L, Go Alan S, Lloyd-Jones, Donald M, Adams Robert J, Berry Jarett D et al. (2011) Heart Disease and Stroke Statistics--2011 Update: A Report From the American Heart Association. Circulation 123:e18-209.

2. Ariza MA, Vimalananda VG, Rosenzweig JL (2010) The economic consequences of diabetes and cardiovascular disease in the United States. Rev Endocr Metab Disord 11: 1-10.

3. Go AS, Mozaffarian D, Roger VL, Benjamin EJ, Berry JD, et al. (2014) Heart disease and stroke statistics--2014 update: a report from the American Heart Association. Circulation 129: e28-28e292.

4. Ely SW, Berne RM (1992) Protective effects of adenosine in myocardial ischemia. Circulation 85: 893-904.

5. Burnstock G (2002) Purinergic signaling and vascular cell proliferation and death. Arterioscler Thromb Vasc Biol 22: 364-373.

6. Berne RM (1980) The role of adenosine in the regulation of coronary blood flow. Circ Res 47: 807-813.

7. Montalescot G (2011) Platelet biology and implications for antiplatelet therapy in atherothrombotic disease. Clin Appl Thromb Hemost 17: 371-380.

8. Fuentes E, Badimon L, Caballero J, Padró T, Vilahur G, et al. (2014) Protective mechanisms of adenosine 5'-monophosphate in platelet activation and thrombus formation. Thromb Haemost 111: 491-507.
9. Cohen MV, Downey JM (2008) Adenosine: trigger and mediator of cardioprotection. Basic Res Cardiol 103: 203-215.

10. Burnstock $G$ (2009) Purinergic signalling: past, present and future. Braz J Med Biol Res 42: 3-8.

11. Cain BS, Meldrum DR, Dinarello CA, Meng X, Banerjee A, et al. (1998) Adenosine reduces cardiac TNF-alpha production and human myocardial injury following ischemia-reperfusion. J Surg Res 76: 117-123.

12. Cohen MV, Downey JM (2011) Ischemic postconditioning: from receptor to end-effector. Antioxid Redox Signal 14: 821-831.

13. McCallion K, Harkin DW, Gardiner KR (2004) Role of adenosine in immunomodulation: review of the literature. Crit Care Med 32: 273-277.

14. Ingwall JS (2009) Energy metabolism in heart failure and remodelling Cardiovasc Res 81: 412-419.

15. Burnstock $G$ (2012) Targeting the visceral purinergic system for pain control. Curr Opin Pharmacol 12: 80-86.

16. Headrick JP, Peart JN, Reichelt ME, Haseler LJ (2011) Adenosine and its receptors in the heart: regulation, retaliation and adaptation. Biochim Biophys Acta 1808: 1413-1428

17. BERNE RM (1963) Cardiac nucleotides in hypoxia: possible role in regulation of coronary blood flow. Am J Physiol 204: 317-322.

18. Ralevic V, Burnstock G (1998) Receptors for purines and pyrimidines. Pharmacol Rev 50: 413-492.

19. Deussen A (2000) Metabolic flux rates of adenosine in the heart. Naunyn Schmiedebergs Arch Pharmacol 362: 351-363.

20. Lloyd HG, Deussen A, Wuppermann H, Schrader J (1988) The transmethylation pathway as a source for adenosine in the isolated guinea-pig heart. Biochem J 252: 489-494.

21. Boison D (2013) Adenosine kinase: exploitation for therapeutic gain. Pharmaco Rev 65: 906-943.

22. Smolenski RT, Suitters A, Yacoub MH (1992) Adenine nucleotide catabolism and adenosine formation in isolated human cardiomyocytes. J Mol Cell Cardio 24: 91-96.

23. Zhang J, Duncker DJ, Xu Y, Zhang Y, Path G, et al. (1995) Transmural bioenergetic responses of normal myocardium to high workstates. Am J Physiol 268: H1891-1905.

24. Hori M, Kitakaze M (1991) Adenosine, the heart, and coronary circulation Hypertension 18: 565-574.

25. Lippe G, Bisetto E, Comelli M, Contessi S, Di Pancrazio F, et al. (2009) Mitochondrial and cell-surface FOF1ATPsynthase in innate and acquired cardioprotection. J Bioenerg Biomembr 41: 151-157.

26. Das S, Steenbergen C (2012) Mitochondrial adenine nucleotide transport and cardioprotection. J Mol Cell Cardiol 52: 448-453.

27. Ertracht O, Malka A, Atar S, Binah O (2014) The mitochondria as a target for cardioprotection in acute myocardial ischemia. Pharmacol Ther 142: 33-40.

28. Dunston CR, Griffiths HR, Lambert PA, Staddon S, Vernallis AB (2011) Proteomic analysis of the anti-inflammatory action of minocycline. Proteomics 11: $42-51$

29. Panayiotou C, Solaroli N, Karlsson A (2014) The many isoforms of human adenylate kinases. Int J Biochem Cell Biol 49: 75-83.

30. Jensen FB (2009) The dual roles of red blood cells in tissue oxygen delivery: oxygen carriers and regulators of local blood flow. J Exp Biol 212: 3387-3393.

31. Dzeja P, Terzic A (2009) Adenylate kinase and AMP signaling networks: metabolic monitoring, signal communication and body energy sensing. Int $J$ Mol Sci 10: 1729-1772.

32. Masuda M, Demeulemeester A, Chen CC, Hendrikx M, Van Belle H, et al. (1991) Cardioprotective effects of nucleoside transport inhibition in rabbit hearts. Ann Thorac Surg 52: 1300-1305.

33. Figueredo VM, Diamond I, Zhou HZ, Camacho SA (1999) Chronic dipyridamole therapy produces sustained protection against cardiac ischemia-reperfusion injury. Am J Physiol 277: H2091-7.

34. Van Belle H (1993) Nucleoside transport inhibition: a therapeutic approach to cardioprotection via adenosine? Cardiovasc Res 27: 68-76. 
Citation: Kolathuru SS, Yeung PK (2015) Therapeutic Potential of Adenosine Transport Modulators for Cardiovascular Protection. Cardiol Pharmacol 4: e127. doi:10.4172/2329-6607.1000e127

Page 3 of 3

35. Isenberg G, Belardinelli L (1984) Ionic basis for the antagonism between adenosine and isoproterenol on isolated mammalian ventricular myocytes. Circ Res 55: 309-325.

36. Yeung $P$, Seeto $D(2012)$ Diltiazem attenuates cardiovascular toxicites and reduces mortality induced by isoproterenol in a freely moving rat model in vivo. Canadian Journal of Cardiology 28:S195.

37. Ford DA, Sharp JA, Rovetto MJ (1985) Erythrocyte adenosine transport: effects of Ca2+ channel antagonists and ions. Am J Physiol 248: H593-598.
38. Rovetto MJ, Ford DA (1987) Inhibition of adenosine transport by verapamil and other calcium channel antagonists. Prog Clin Biol Res 230: 223-233.

39. Li RW1, Yang C, Sit AS, Lin SY, Ho EY, et al. (2012) Physiological and pharmacological roles of vascular nucleoside transporters. J Cardiovasc Pharmacol 59: 10-15.

40. Yeung $P$, Seeto $D$ (2013) A study of the effect of isoproterenol on red blood cell concentrations of adenine nucleotides in a freely moving rat model in vivo. Cardiovas Pharmacol (Open Access) 2:102. 